\title{
QRcode, hashtag or audio watermark? A case study on second screening
}

\author{
Alan César Belo Angeluci ${ }^{1}$ - Gustavo Moreira Calixto ${ }^{2}$. \\ Leire Mara Bevilaqua ${ }^{3}$ - Gleice Bernardini ${ }^{4}$. \\ Maria Cristina Gobbi ${ }^{4}$
}

Received: 16 July 2015 /Revised: 10 February 2016 / Accepted: 29 February 2016 /

Published online: 11 March 2016

(C) Springer Science+Business Media New York 2016

\begin{abstract}
Combining television and a second, web-connected screen, is challenging both in technical and production content issues, as they can influence users' engagement with the content. The case study presented in this paper aims to discuss how the use of different content recognition techniques for TV set and mobile devices synchronization can impact for better or worse the users' interactive experience. From a triangulation method approach, using a prototype, quantitative and qualitative questionnaires and focus groups, user test sessions were carried out in order to collect perceptions and reactions from participants using variations of QRcode, hashtag and audio watermark markers when second screening. Data analysis showed that a key factor for a fruitful content recognition for dual screening is not only the seamless
\end{abstract}

\author{
Alan César Belo Angeluci \\ aangeluci@uscs.edu.br \\ Gustavo Moreira Calixto \\ gustavo.mcalixto@sp.senac.br \\ Leire Mara Bevilaqua \\ leire@tvu.unesp.br \\ Gleice Bernardini \\ gleicebernardini@hotmail.com \\ Maria Cristina Gobbi \\ mcgobbi@faac.unesp.br
}

1 Programa de Pós-Graduação em Comunicação, Universidade Municipal de São Caetano do Sul, USCS, Rua Santo Antônio, 50, Centro, 09521-160 São Caetano do Sul, SP, Brazil

2 Centro Universitário Senac - Santo Amaro, Avenida Engenheiro Eusébio Stevaux, 823, Santo Amaro 04696-000 SP, Brazil

3 Televisão Universitária UNESP, Rua Jacy Stevaux Villaça, 2-99, 17047-250 Bauru, SP, Brazil

4 Programa de Pós-Graduação em Comunicação, Universidade Estadual Paulista, UNESP, Avenida Engenheiro Luiz Edmundo Carrijo Coube, 14-01, 17033-360 Bauru, SP, Brazil 
communication by itself, but the influence level of each specific characteristics of a marker, such as size of images, number of characters and noises.

Keywords Second screen $\cdot$ TV $\cdot$ QRcode $\cdot$ Hashtag $\cdot$ Audio watermark Content recognition

\section{Introduction}

Second screeners became an interesting profile to be further investigated in the new media domain. When it comes to watch television and interact in the same time with digital mobile devices, they coordinate their attention between looking for more information in websites and social media, discussing with friends and unknown people, interacting with synchronous and asynchronous content and assimilating the audiovisual object from the TV program.

Digital media devices can take advantage of several communication protocols, such as broadcast and broadband transmission, so they can be used for enabling a bidirectional flow bridge to connect users, content and platforms. Second screen markers are an effective way to make it work and fundamental objects of any interactive application in this landscape. They need to be less disruptive as possible, making it a seamless process for the users. An interesting approach considers content recognition techniques using audio, video, applications and many other sources for triggering an action when dual screening.

QRcodes, hashtags and audio watermarks can be listed as markers known and largely used by the market for content recognition in second screen experiences. The case study presented in this paper aims to analyze the impact of variations of these markers in the user's perceptions and reactions. It can gives some traces for TV producers and Internet content providers, eager to understand better this swiftly changing television environment. The next sections described some literature and scholars' perspective for this case study, as well as the methods, description of tests, analysis and generated outputs.

\section{Context}

Second screening experiences have revealed how the intensive use of converged multiple platforms is strongly part of individuals' daily activities. New media technologies for second screen became integrated into social relationships and structures and are not only restricted to particular experiences in households and domestic environments [16], but in a wider variety of spaces and consuming units. However, it is worthy pointing that second screeners are much more than consumers, but prosumers immersed in a participatory culture in which they interact, discuss, create, share and feel social connected $[6,20]$.

Scholars describe media experiences as a choice that users make to reach certain individual needs [21]. Grounded on the idea of Rubio et al. [14], it is possible to assume that second screen experiences are based on interactive processes that may occur in some different forms.

A second screen experience can be considered from a social TV perspective: when users establish a relation between a TV event and a social media for reading, searching, producing, sharing, relating and creating content. The function of Facebook and Twitter has been widely described by many authors $[9,15,25]$, and show how information can be gathered from the public, what can influence viewers' attitudes and opinions and which can be the most or least engaging moments in the TV program storytelling. 
Thereafter, second screen experiences can be a result of dedicated applications: when users are stimulated to interact synchronous or asynchronously with an application developed exclusively for a TV program - such as in the case of Hannibal's TV series or the Brazilian SuperStar musical reality $[1,7]$. In both cases, the audience can be engaged with different levels of immersion, merging individual with collective interactions, combining different tools and media and experiencing a more sophisticated act of watching TV with unique characteristics.

Second screen experiences tend to provoke the engagement of different age ranges. In fact, while watching TV, people aged over 34 years old tends to use second screen for information seeking, and millennials prefer to use it for discussing topics in social networking sites [15].

If, in one hand, second screening creates opportunities of new business models and new digital content formats, in the other hand the use of combined platforms concerns broadcasters and Internet content providers, since the attention focus may provoke a temporary or permanent migration to one specific platform while interacting. Several studies have investigated this matter $[5,17]$.

This proper functioning of platforms and applications enabling an efficient second screen experience is also a relevant factor for the entire process. In order to make the experience as seamless as possible, research related to the use of content recognition markers should focus on trialing these techniques and obtaining feedback from final users for improving the experience.

\subsection{Related works}

Literature has shown a variety of studies regarding multiple screens in the general case. In the specific topic of second screen content recognition, there are concerns both in technical and user experience aspects, but usually these approaches are considered separately. In [10], authors developed a QRcode to be detected by user devices for an over-the-top (OTT) platform. Another similar study dealt with the use of an HbbTV interactive TV platform [26]. However, no tests considering the user experience were carried out in both cases. Regarding studies with hashtags, use habits and behaviors and other social aspects issues for final users are a more common approach $[11,12]$.

Audio watermarking has a wide spectrum of application in consumer electronic market, such as for copyright protection [4], monitoring [22], fingerprinting, indication of content manipulation and information carrier [2]. In the second screen landscape, audio fingerprint and audio watermarks are used as a feature for an automatic content recognition (ACR) [23]. Research in this subject is more focused in technical issues [13, 19]. Technically, ACR may give considerable advantage in the synchronization process, but some social aspects must to be considered in order to give a more complete parameter.

The next sections present the case study development approach and the empirical assessment of impact in the user's behavior and social aspects, when dealing with different markers for content recognition in second screen experiences, embodied by prototypes.

\section{Research questions and method}

The hypothesis considered in this case study was the seamless communication as a key factor for a positive perception and reaction of a second screen experience: the less steps are required for the user to interact, the better and more fluid is the second screen experience. Nevertheless, 
content recognition markers characteristics may have influence in the process. The study was so conducted under the following four research questions:

1. QRcodes, hashtags and audio watermarking: which one seems to have more positive aspects for a seamless second screen experience?

2. How differences in the size of the QRcode affect user's second screen interaction?

3. The number of characters in hashtags is a relevant constraint when using it for a second screening action?

4. Are the audio watermark techniques less disruptive and applicable in many sorts of second screen scenarios?

A triangulation method (Fig. 1) was conceived in order to create a guideline for user test sessions, and comprised the simulation of experimenting different improved second screen scenarios, the step-by-step evaluation and the analysis of users' perception and reaction. Low-tech prototype, quantitative and qualitative tools and focus group technique were used in a combined way to generate satisfactory outcomes.

\section{User's test}

Tests were carried out in a soundproof test room with cameras for audio and video recording to support data analysis process. Chairs where participant sat were placed in a semicircle with a radius of 1.5 times the diagonal width of the TV (Fig. 2), as recommended by TV manufactures and department stores [3, 18, 24].

For the tasks using QRcodes, hashtags and audio watermarks, a low-tech second screen prototype was developed containing a 2-min video excerpt of a TV news program; different content recognition markers were inserted using standard video edition software. Five (05) mobile devices were prepared to be used by participants during the test: one (01) Android OS tablet, two (02) Android OS smartphones, one (01) Windows Phone OS smartphone and one (01) MacBook - all capable to connected in a local Wi-Fi network and with a HTML5 compatible browser available. A Raspberry Pi Model B single board was used to play the media, transmitted through a HDMI cable to a 50-inch TV set positioned inside the test room as illustrated in Fig. 2. Moreover, mobile devices were prepared with a pre-installed

Fig. 1 Triangulation method approach for user's test

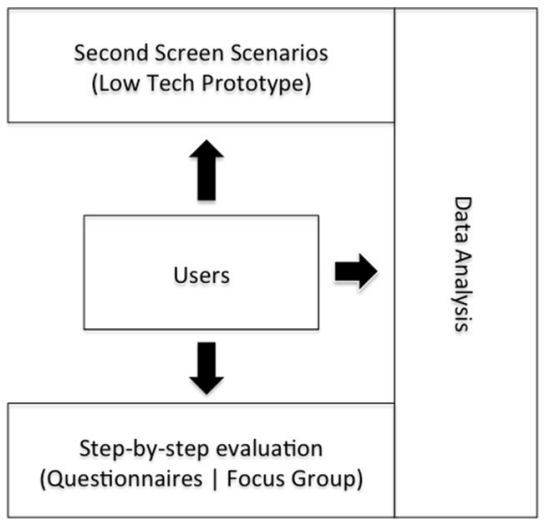


Fig 2 TV and users positioning

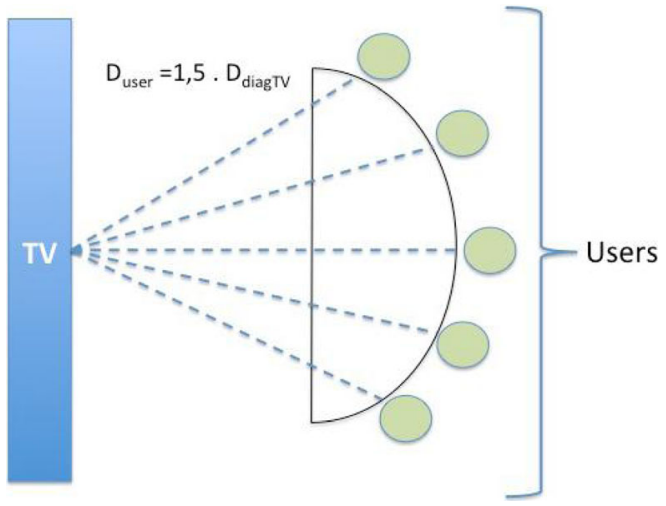

application for QRCode reading and a web browser for hashtag search and to invoke the audio watermark application, following minimal software requirements as follows:

- QRCode: application able to capture QRCode image interested area, proceed with reading process and providing an ASCII character string. For this experimental setup, it is expected an URL to be opened using a web browser.

- Hashtag: a web browser able to provide search tools to link hashtags to a content provided in the Internet cloud.

- Audio watermark: a web browser able to run HTML5-based applications to capture audio, do a parsing process to find information (a ASCII character string) and link to an event in the software front-end. Even if this web application has the feature to detect and trigger events from audio watermark messages, it is a pre-condition an audio sensor allowed to be used and, moreover, a compatibility hardware to detect an audio frequency rate.

The experiment was carried out considering different second screen scenarios with variables for each marker: a $25 \mathrm{~cm}^{2}$ QRCode (called "bigger" during user test sessions, shown in the screen on the top of Fig. 3) and a $12 \mathrm{~cm}^{2}$ QRCode (called "small", shown in the screen on the bottom of Fig. 3), leading to the same website. It expects a variation of QRCode reading performance related to size variation [8].

Also, a 12-characters hashtag expression (shown in the screen on the top of Fig. 4) and a 6-charaters one (shown in the screen on the bottom of Fig. 4), were considered to be typed in the browser. Although general guidelines shows that typing less is easier and more recommended, a written language may varies its meaning depending on the idiom used. Easterner's languages, for example, may be very effective with two words or symbols, but some Latin languages demands more letters to be clearly understood. Sometimes small words can be so generic and without specifying their content it may cause doubts. This was considered due to the participant's profile.

Two audio watermark frequencies were used: one very possible to be heard by humans $(12,500-13,000 \mathrm{~Hz}$ ) and a second one in the limit to be inaudible (about $18,000-18,500 \mathrm{~Hz}$ ), both played when an icon is presented in the top of TV screen (Fig. 5). Two different audio configurations are used to verify both user experience and acceptation perspective considering audible and inaudible frequency rates. A single tone with multiple frequencies message was used, representing the word 


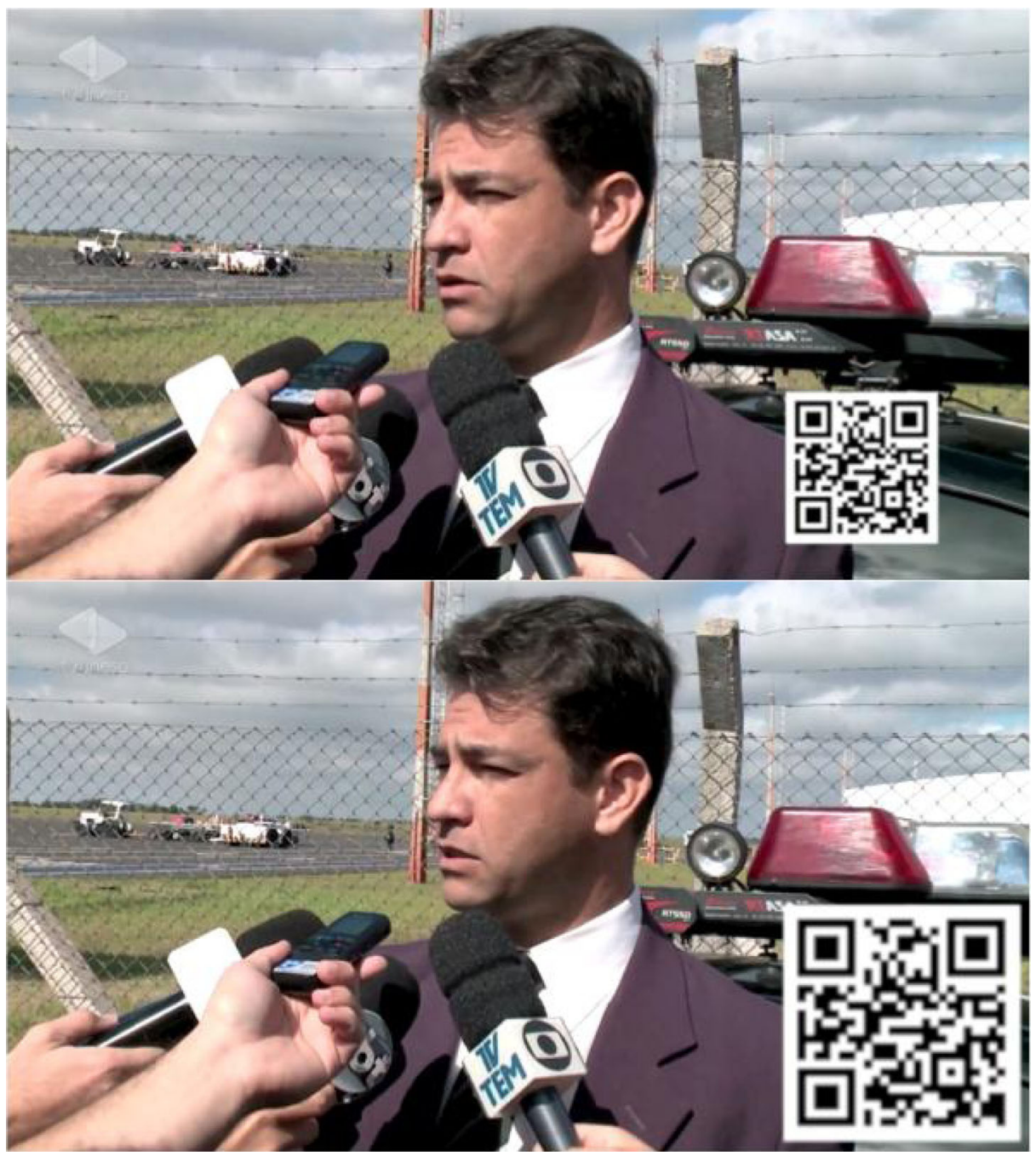

Fig. 3 QRcode experiment on the TV screen

"TV". The second screen application was calibrated to recognize messages in both frequency ratings. A specific study with audio watermark was carried out in [8]. This experimental setup is shown on Fig. 6.

Participants chosen were young people aged between 20 and 36 years old, with regular use of mobile devices and interest in watching TV. In total, 15 users were part of this case study in order to collect qualitative data. Participants selected were randomly distributed in three separated sessions in groups of 5 people. Each test session were carried out under the following test protocol, divided in three stages:

- Firstly, questionnaires were applied in order to collect information on uses and habits related to TV, mobile devices and second screening. 


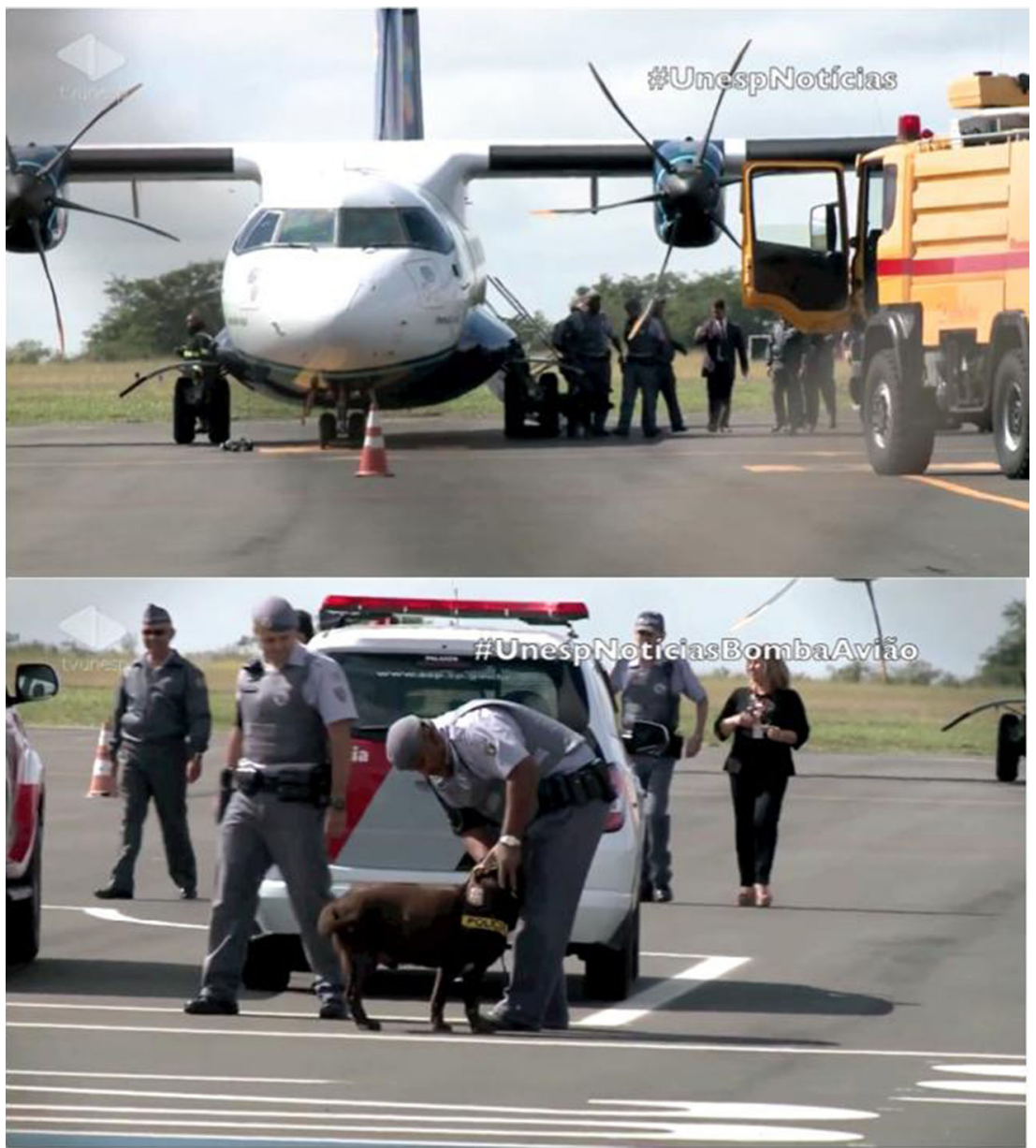

Fig. 4 Hashtag experiment TV screen

- Secondly, participants were stimulated to interact and engage in second screen tasks using TV and mobile devices provided, considering the two different scenarios for QRcode, hashtag and audio watermark tags described above. In the end of each round, users were requested to answer a new survey with staggered and open-ended questions.

- Finally, a focus group was conducted based in semi-structured questions and situations observed by the moderator along the test, aiming to uncover issues not considered before, and going deeper in topics emerged by participants during the group discussions.

In all cases, the markers were shown for $10 \mathrm{~s}$ while running the video excerpt, in the same stretch. They led the users to websites related to the content being displayed on TV during the interactive moment. The test moderator never aware participants about the different scenarios and stimulated them to comment, move around the room and perform all actions they may consider necessary to achieve the second screen experience (e.g., stand up from the chair, get closer to the TV set, manipulate the applications available in the mobile devices, etc.). 


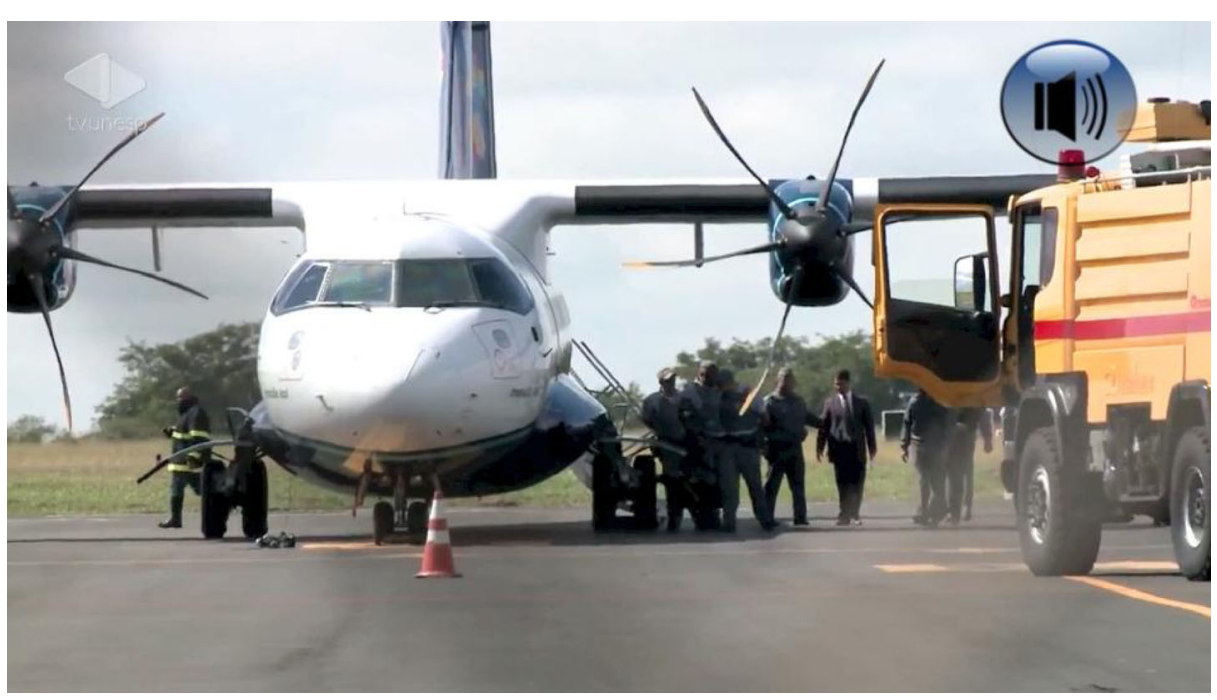

Fig. 5 Audio watermark experiment TV screen

\subsection{Data analysis}

As not all devices used were from the participants, it is worthy pointing that there was some expected difficulties by users in dealing with the device applications. In the first session, one of the participants had no familiarity with Windows Phone OS and could not manage to use the phone in any tasks. Considering this, the moderator gave an extra time for participants to get more confident with the devices in the next sessions, which seems to had solved the problem, although few users still had difficulties and got frustrate and upset.

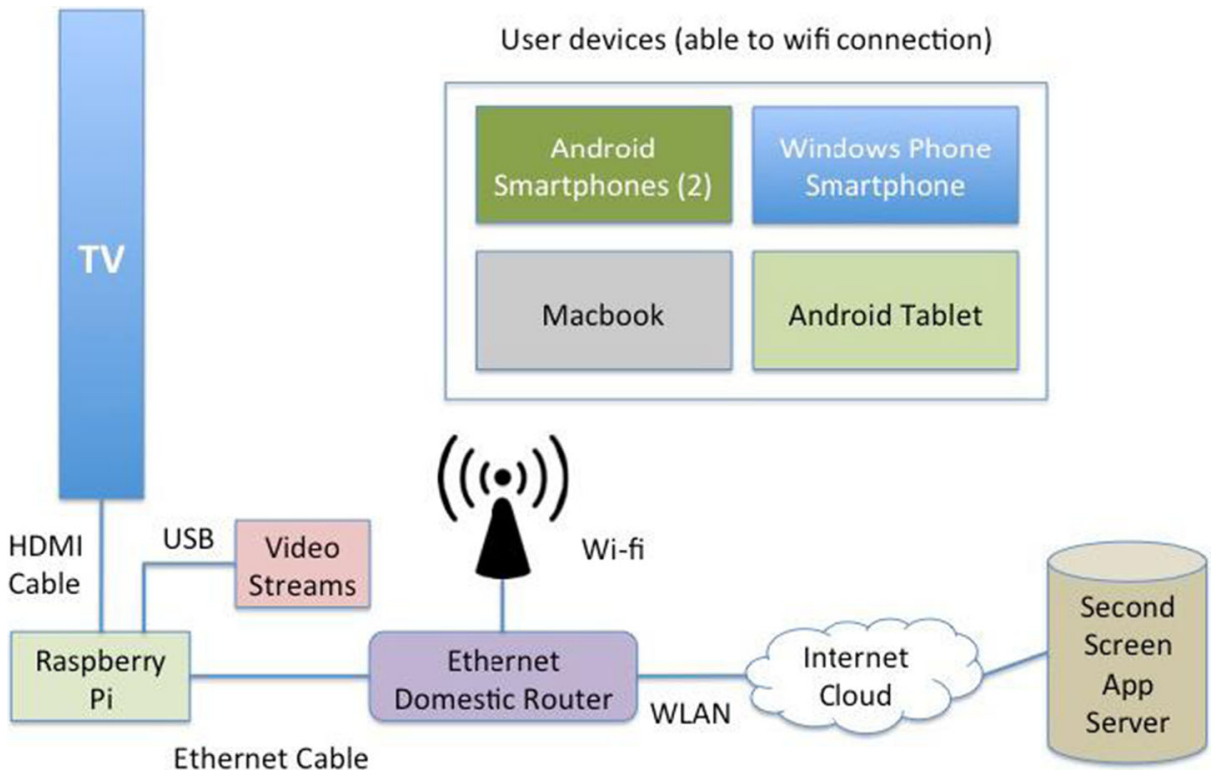

Fig. 6 User test devices setup 
All 15 participants mentioned having mobile devices and TV set in their home, and two said to have very simple phones, without Internet connection. Regarding the TV possession, there were a wider variety of types, from CRT to LCD flat panel and smart TVs. Notebooks and tablets are part of their daily activities with a satisfactory penetration.

When asked about second screening activities between TV and mobile devices, 9 of 15 mentioned to know the experience but never had one before. Also reported having performed actions such as (1) commenting and posting in social media about a subject being shown on TV at that time, (2) accessing an online content while watching a TV program due to a hashtag provided by the broadcaster, and (3) searching for information in browser search engines during the transmission of a TV program. This reveals that, for this specific group, the second screen experience is still more linked to the social TV experience than the proper second screen concept. The other six participants mentioned to have had one or more second screen experiences before, such as interacting in real time with a TV program storytelling using an application in a mobile device, with content automatically updated during the TV program.

When asked about a general rate for their second screen activities, the overall average rate was 7.3. The second screen experience is interesting in most of the time for 11 participants, and for 13 of them a TV program would call more attention if they explored more the interactivity of a second screening task. Worthy pointing that for the other two people, the second screen activity is something that hinders the watching TV experience.

\subsubsection{QRcode: the bigger, the better - but not too much}

Users tend to prefer the $25 \mathrm{~cm}^{2}$ QRcode size when using them for second screening activities (Fig. 7). The $25 \mathrm{~cm}^{2}$ image is better for 7 interviewees that strongly agree/agree with the statement (4 participants strongly disagree/disagree, the same number of people that are neutral). This was also confirmed as 12 respondents totally disagree/disagree when asked if they had more difficulties in managing with the $25 \mathrm{~cm}^{2}$ QRcode image. The overall rate for the $25 \mathrm{~cm}^{2}$ QRcode image experience was 6.8; for the $12 \mathrm{~cm}^{2}$ image, 4.6. Test sessions showed that participants could not easily position the mobile devices in front of the TV screen to scan the $12 \mathrm{~cm}^{2}$ QRcode image codes. Difficulties were related mainly in relation to the distance between chairs and TV set, the angle and focus of the mobile device camera lens, requiring them to stand up and put the mobile device too close of the TV screen, many times with little success. All this process is time consuming, and 10 of 15 users felt they lost less time in trying to scan the $25 \mathrm{~cm}^{2}$ QRcode image.

Focus group discussions revealed that participants are friendly to the use of a QRcode for a second screen experience as, in general, it requires less steps to achieve the goal and is "very easy and practical", according to their feedback. However, a big size requires attention as it may covers or blurs relevant audiovisual content being transmitted by the broadcaster. Also, the 10 -s time provided for scanning the image was not enough for 7 participants, which brings to the attention/disruption issue: although QRcodes were well evaluated, 10 respondents claimed they lost the focus on the TV program due to the interactive action with the QRcode.

\subsubsection{Hashtags: less typing, please}

Participants preferred concise and creative hashtags, as 11 respondents reported to be more favorable to the 6-characters hashtag. The overall rate for the 12-characters hashtag second screen experience was lower (2.5) if compared to the 6-characters (5.7). 

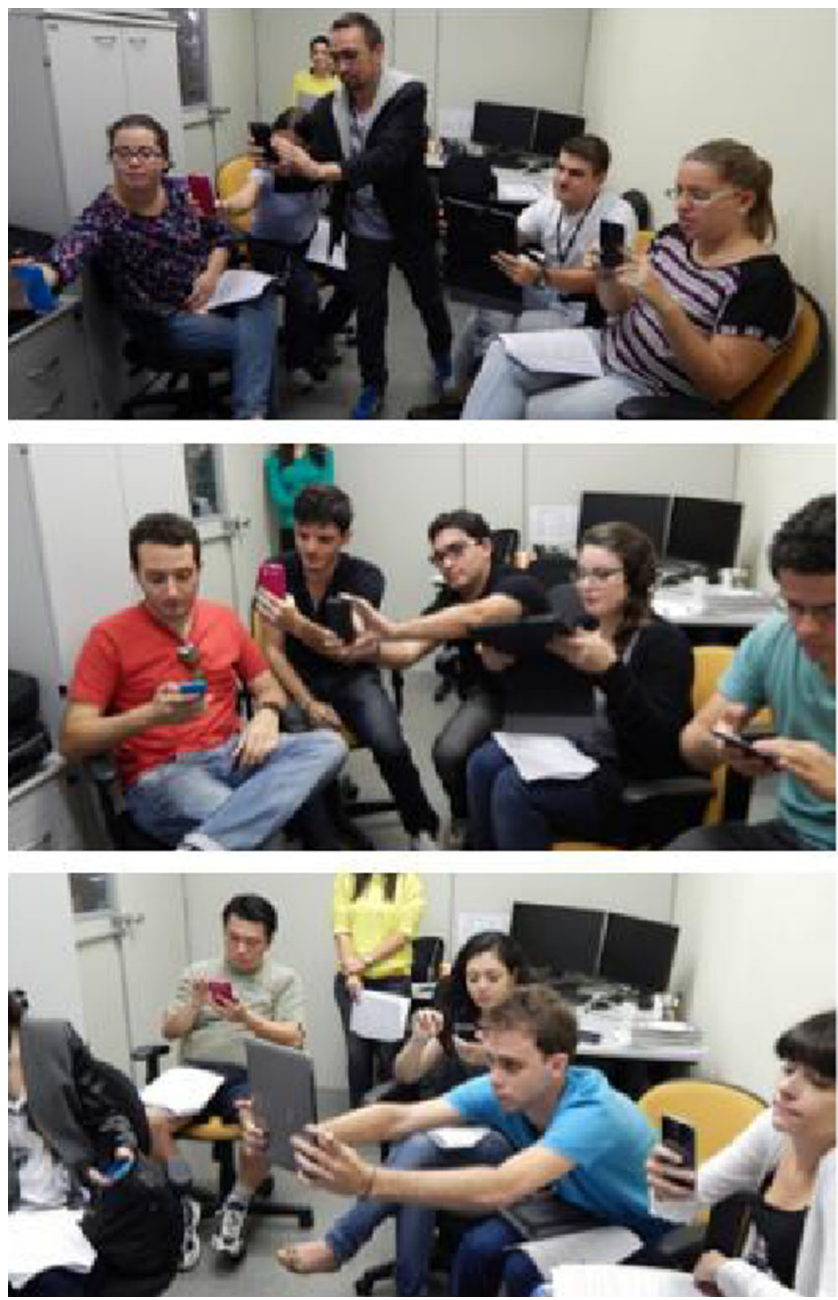

Fig. 7 Groups of participants scanning QRcodes during test sessions

Users complained about the process of typing too many characters and keep up with the TV content at the same time, and 13 interviewees founded harder to find content using the bigger hashtag. All 15 users disagreed with the sentence "I quickly accessed the hashtag content equally in both situations". The attention/disruption issue was also remarkable again: 14 users observed they had lost information from TV content when typed the hashtag, and ten mentioned that hashtags are more disruptive than QRcodes when second screening. Figure 8 illustrates users typing a hashtag.

This reinforces the hypothesis of the seamless communication as a key factor for a better second screen experience, but also considering specific aspects: a 12-characters hashtag is more disruptive than a 6-characters one, which is worse than a QRcode. From 15 users, nine considered the experience with QRcode faster and with less steps. It is worthy pointing that for testing purposes, markers were applied in a specific use scenario; it is not possible to assume that a QRcode is always more effective. Curiously, the TV market seems to explore much less 


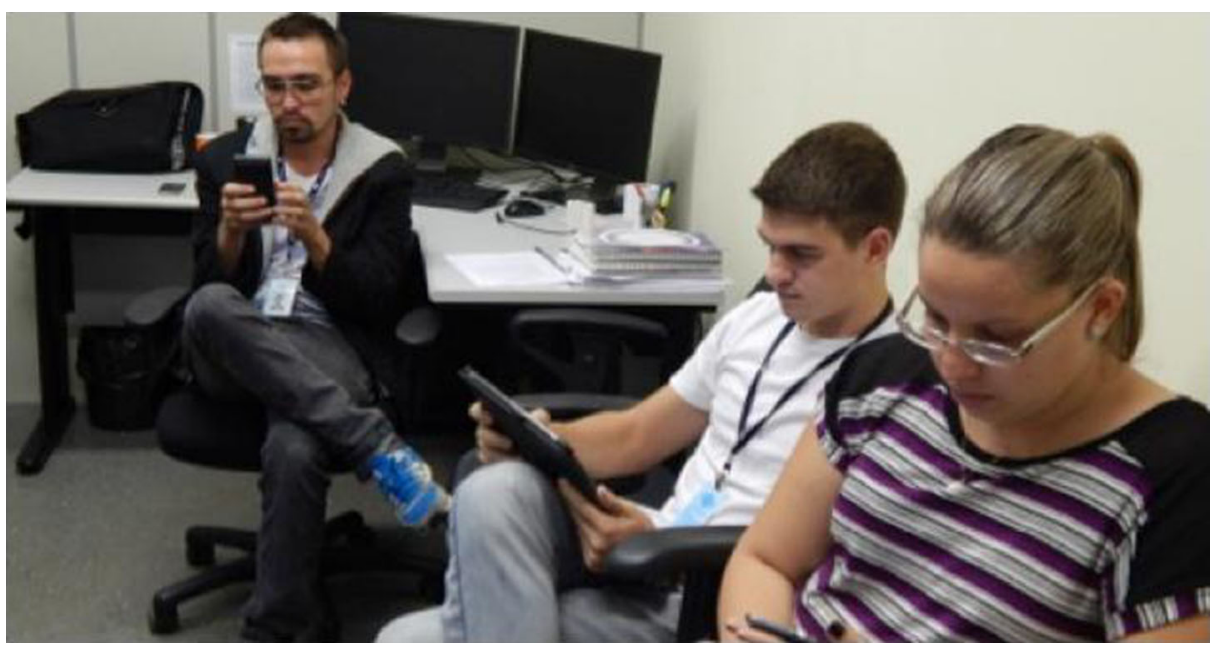

Fig. 8 Users totally focused in typing the hashtag

QRcodes for second screen experiences than hashtags, which seems to be leveraged due to the high use of Twitter and other social networks.

\subsubsection{Audio watermark: noises are knockout}

The frequency used in the audio watermark rounds were both audible by humans in different sound levels, but the audio watermark used in the second round was louder, using a $1800 \mathrm{~Hz}$ frequency. Although noise might be annoying, mainly when there is a main layer of audio in the test room (in this case, the TV video excerpt), the number of respondents neutral to the variation of this noise was five, compared to six users that rejected the $1800 \mathrm{~Hz}$ frequency audio watermark. The interactive process was not easy in both situations for 11 participants and neutral for the rest. Figure 9 presents users trying to capture audio watermark. The overall average rate for the $12500 \mathrm{~Hz}$ frequency audio watermark was 4.4 and for the $18000 \mathrm{~Hz}$ was 3.6.

Focus group showed that one of the main problem when interacting with audio watermark was a mobile device restriction: most of the mobile devices did not properly captured the wave frequency at the very first moment. The lower is the frequency, the easier is for the device microphone to recognize the waves - but the louder is the tune noises for human hearing.

If, in one hand, the audio watermark seems to be the most seamless communication process - as it only requires the device to identify the frequency waves emitted from TV to start the mobile device content - in the other hand, any level of sound from the noisy tunes are critical and disruptive, even the lower ones. The statement "the noise sound in the first experience (lower noise) do not disturbed the experience" was disagreed by seven participants and neutral by others six.

When comparing the use of audio watermark, QRcode and hashtag, opinions were diverged: seven participants preferred audio watermark to QRcode and hashtag, six think the opposite and two respondents were neutral. Part of the group was strongly influenced by the annoyance of the audio watermark tune noises. Comments during the focus group also reveal that the noise make it harder to hear the audio from TV program, suggesting if a new 


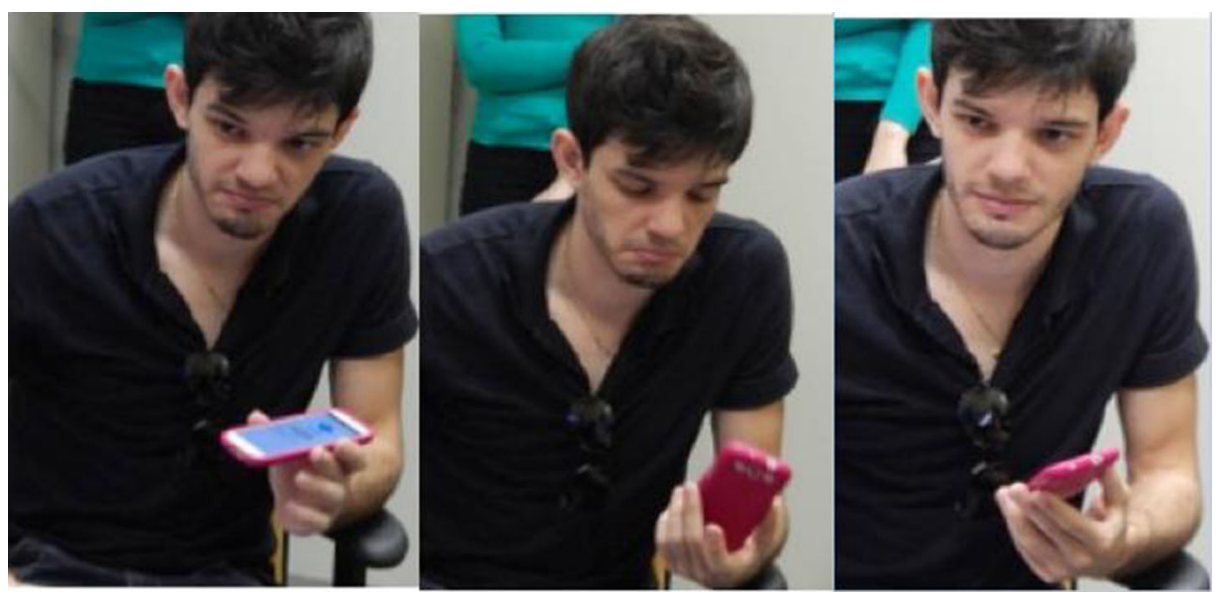

Fig. 9 User trying to capture audio watermark

frequency inaudible for humans would be used, this technique would be the most adequate for second screening. Another suggestion by participants was to run an audio watermark as a reminder for the user to decide whether to interact or not with a coming second screen activity.

\section{Final remarks and future works}

Summing up, audio watermark and QRcode presented less number of steps for second screening and were considered the more adequate markers for an improved experience, as they both provided better seamless communication. Although it reinforces the trend towards the ACR, they are more likely to equipment restrictions that may interfere in a fruitful interaction. Considering the study case, hashtags are the less indicated for a proper second screen improved experience; although they give more freedom for users to control the process, it requires more effort in typing and consequently reduce the attention on TV content, reducing significantly the number of possible interactive actions during a TV program. Although it might seems a clear guideline for some experts, TV broadcast market also seems not to be really aware and fully in line with these known guidelines, as it is possible to verify one or other TV show still using very big hashtags for second screening.

QRcodes images provided a fast and efficient synchronization, but strongly depend on certain efforts from users if the mobile camera lens is not adequate to scan the codes rapidly and properly. When using QRcodes images, sizes forcing users to execute too many movements or blurring the audiovisual content should be avoided.

Regarding the audio watermarks, user test sessions in the future would benefit a deeper analysis of this technique, as a human inaudible frequency would lower the negative impacts of tune noises in the interaction process; a more accurate requirements list for equipment used in test could also avoid bad functioning in the process of wave's frequency recognition. Last, but not least, further studies should consider relevant contents to be provided in the second screen, aligned in a creative and engaging way to the TV content, as participants reported they would have had a more immersive second screen experience if the content in the mobile device was dedicated to the interactive action. 
In additional, this case study pushed up new topics to be investigated regarding TV and second screen experience. First of all, user tests could be expanded to other environments, for instance, public spaces and other environments beyond a traditional leisure context, considering some new variables to be taken into account (noise, illumination conditions, etc.). Moreover, a quantitative analysis to measure performance indicators, including more variances of tags, TVs, second screen application, devices and other relevant aspects should contribute to improve the interactive experience reliability.

\section{References}

1. Angeluci ACB (2013) From 'Gads' to 'Apps': the key challenges of post-web internet era. Revista Geminis 1(2 Ano 4):75-88

2. Arnold M (2000) Audio watermarking: features, applications, and algorithms. IEEE Int Conf Multimedia Expo (II) 1013-1016

3. Bestbuy TV buying guide. Available at http:/www.bestbuy.com/

4. Boney L, Tewfik AH, Hamdy KN (1996) Digital watermarks for audio signals. In: Multimedia Computing and Systems, 1996, Proceedings of the Third IEEE International Conference on. IEEE 473-480

5. Brown A et al (2014) HCI over multiple screens. In: CHI'14 Extended Abstracts on Human Factors in Computing Systems. ACM 665-674

6. Bruns A (2008) Blogs, Wikipedia, second life, and beyond: from production to produsage. Peter Lang

7. Caldas CHS, De Oliveira BJ, Neto ON (2015) Dos regimes aos gradientes de interação no consumo televisivo: superstar, um reality show nascido para ser interativo. Verso Reverso 29(70):24-34

8. Calixto GM et al (2014) Effectiveness analysis of audio watermark tags for IPTV second screen applications and synchronization. In: Telecommunications Symposium (ITS), 2014 International. IEEE 1-5

9. Cameron J, Geidner N (2014) Something old, something new, something borrowed from something blue: Experiments on dual viewing TV and Twitter. J Broadcast Electron Media 58(3)

10. Das D et al (2011) An interactive system using digital broadcasting and quick response code. In: Consumer Electronics (ISCE), 2011 I.E. 15th International Symposium on. IEEE 397-400

11. Doughty M, Rowland D, Lawson S (2011) Co-viewing live TV with digital backchannel streams. In: Proceedings of the 9th international interactive conference on Interactive television. ACM 141-144

12. Doughty M, Rowland D, Lawson S (2012) Who is on your sofa?: TV audience communities and second screening social networks. In: Proceedings of the 10th European conference on Interactive TV and video. ACM 79-86

13. Duong NQK, Howson C, Legallais Y (2012) Fast second screen TV synchronization combining audio fingerprint technique and generalized cross correlation. In: Consumer Electronics-Berlin (ICCE-Berlin), 2012 I.E. International Conference on. IEEE 241-244

14. Galindo RF, Fernández BE, Alameda GD (2014) Análisis del uso e interacción con aplicaciones second screen en TV Social en España. Cuadernos Inf (35)159-175

15. Giglietto F, Selva D (2014) Second screen and participation: a content analysis on a full season dataset of tweets. J Commun 64(2):260-277

16. Hirsch E, Silverstone R (Ed.) (2003) Consuming technologies: media and information in domestic spaces. Routledge

17. Holmes ME, Josephson S, Carney RE (2012) Visual attention to television programs with a second-screen application. Proceedings of the Symposium on Eye Tracking Research and Applications. ACM 397-400

18. How to choose the right screen size. Available at http://www.amazon.com//

19. Howson C et al (2011) Second screen TV synchronization. In: Consumer Electronics-Berlin (ICCE-Berlin), 2011 I.E. International Conference on. IEEE 361-365

20. Jenkins H (2009) Confronting the challenges of participatory culture: media education for the 21 st century. Mit Press

21. Katz E, Blumler JG (1999) GUREVITCH, Michael. Utilization of mass communication by the individual. Sources notable selections in mass media, p. 51-59

22. Lemma A et al (2008) Watermarking for content aware intelligent toys. In: 2008 Second International Conference on Electrical Engineering

23. Lexicon: Second Screen Society. Available at http://www.2ndscreensociety.com/lexicon/

24. The right distance to sit from your TV. Available at http://www.lg.com// 
25. Wohn DY, Eun-Kyung NA (2011) Tweeting about TV: sharing television viewing experiences via social media message streams. First Monday (16)3

26. Ziegler C (2013) Second screen for HbbTV - automatic application launch and app-to-app communication enabling novel TV programme related second-screen scenarios. In: Consumer Electronics Berlin (ICCEBerlin), 2013. ICCEBerlin 2013. IEEE Third International Conference on. IEEE 1-5

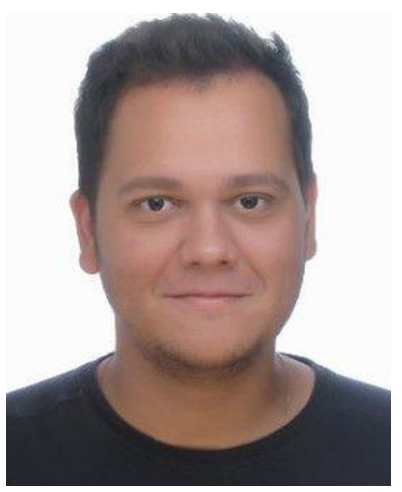

Alan César Belo Angeluci holds a Ph.D. in Sciences from Polytechnic School at the University of São Paulo, (USP), Brazil, with sandwich period at University of Brighton, England; assistant professor at Master's Program in Communication and Innovation from Municipal University of São Caetano do Sul, USCS; he carried out his post-doctoral period at The University of Texas at Austin, USA. Research leader of Smart Media \& Users Research Group. He also serves as the reviewer of refereed international magazines and journals among others.

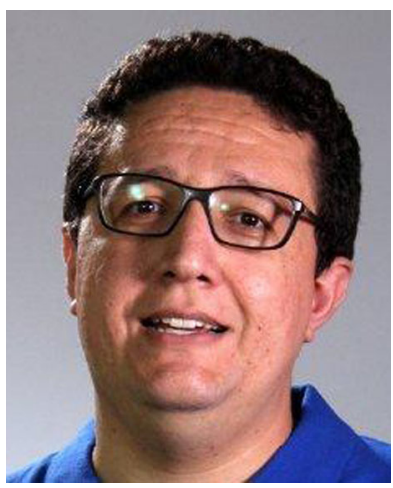

Gustavo Moreira Calixto holds a Master in Electrical Engineering from University of Campinas and a Ph.D. in Sciences from Polytechnic School at the University of São Paulo (USP), Brazil; he was a visiting researcher at The University of Texas at Austin, USA. He is assistant professor at the University SENAC, Brazil. Researcher at the Interdisciplinary Center for Interactive Technologies at the University of São Paulo. He is also a volunteer at IEEE. 


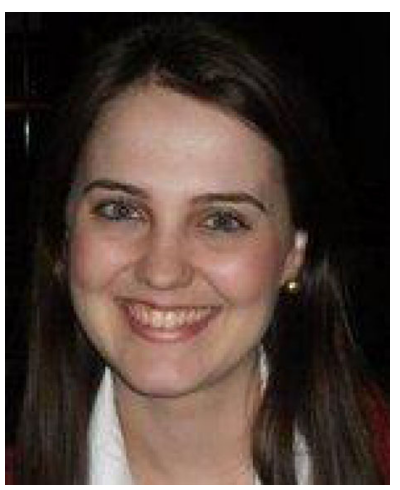

Leire Mara Bevilaqua holds a Master in Digital Television from University of State of São Paulo (UNESP) and is a $\mathrm{PhD}$ candidate at the same institution. She is member of Audiovisual Studies Research Group and works at UNESP TV station.

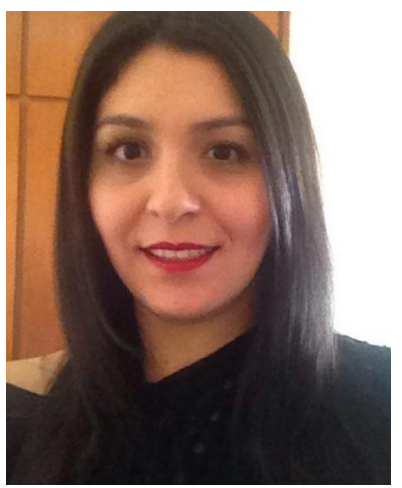

Gleice Bernardini holds a Master in Communication from University of State of São Paulo (UNESP) and is specialist in Language, Culture and Media. 


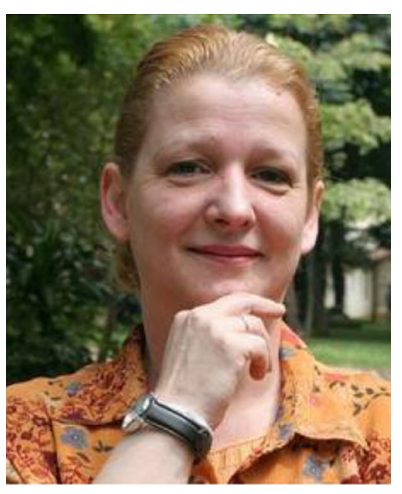

Maria Cristina Gobbi holds a Ph.D. in Communication from the University of São Paulo, (USP), Brazil; assistant professor at $\mathrm{PhD}$ and Master's Program in Communication and vice-coordinator of Master's Program in Media and Technology from University of State of São Paulo (UNESP); She serves as the reviewer of refereed international magazines and journals among others. 